Vol. 6, Num. 1, 2021

\title{
SOFTWARE SYSTEM OF DECODING LIGHT CODES
}

\author{
Vladyslav Barsiienko, Oksana Lashko \\ Lviv Polytechnic National University, 12, Bandera Str, Lviv, 79013, Ukraine. \\ Authors' e-mail: vladyslav.barsiienko.ki.2017@lpnu.ua
}

https://doi.org/10.23939/acps2021.01.

Submitted on 01.05.2021

(C) Barsiienko V., Lashko O., 2021

\begin{abstract}
The paper presents the stages of creating a software system for decoding light codes and examines the effectiveness of its operation. The relevance of the development is due to the need to combine the speed and distance of information delivery for the further development of wireless communication systems.

The authors' attention is focused on considering the basic components of the designed system. The main algorithms of operation have been presented and the effectiveness of their development has been proved. A comparison with existing means has been made and the prospects for the development of cable free optical communication systems have been emphasized.
\end{abstract}

Index Terms: .NET Framework, C\#, Windows Forms, Light signals, Morse, Optical communication system, Wireless optical communication

\section{INTRODUCTION}

Today, modern people use various types of communication and information transmission on a daily basis. This helps to reduce the time spent on routine tasks, work communication, and news dissemination. Information transmission also plays an important role in the scientific and research spheres, because the faster scientists receive data from the depths of the Earth or deep space, the faster it is distributed in the scientific community - the faster and better they will be able to make discoveries.

Fiber-optic networks have already been actively used within the Earth, where information is transmitted in the form of light signals, and the only delay is created by the transformation of a light carrier into an electric one, but the system has a significant factor that hinders its development: unlike electromagnetic waves, we have not yet learned how to use the wave nature of light in a controlled way, which does not allow us to transmit information without a cable with a light beam [1]. But technology does not stand still, something that we cannot use at long distances within our atmosphere - it can be used at short distances or unlock the limitless potential of cable-free transmission of information by light signals outside the Earth.

The implementation of a light code decoding system requires a thorough study of light as an information carrier, its weaknesses and features. Where among the important ones: the behavior of light in different environments, Redshift.

To develop this system, one needs to correctly approach the development of key modules, including: intercepting the image from the camera, converting the light signal into Morse code, converting Morse code into readable text.

The practical result of performing The Bachelor's work is the implementation of a system that will demonstrate the prospects of cable-free information transmission systems based on light codes.

\section{USAGE OF LIGHT AS DATA CARRIER}

Today, people have discovered many algorithms and methods for storing and transmitting information. Starting from clay tablets and ending with flash drives based on DNA molecules. Understanding the nature of physical phenomena, humanity has learned to apply them when they need it and how they need it. Until recently, electric current served as the power of everything that surrounded us: work of electrical appliances, changes in the electrical parameters of the body indicate diseases, in cables between houses, current carries information, text, voice, images. We have mastered current enough, but it's time to go further, progress does not stand still, new technologies need more reliability, higher speed.

Light has become a new promising information carrier [2]. People have never fully understood its nature and properties, but now, our knowledge is enough to start applying it, to expand our vision of future technologies with a new branch.

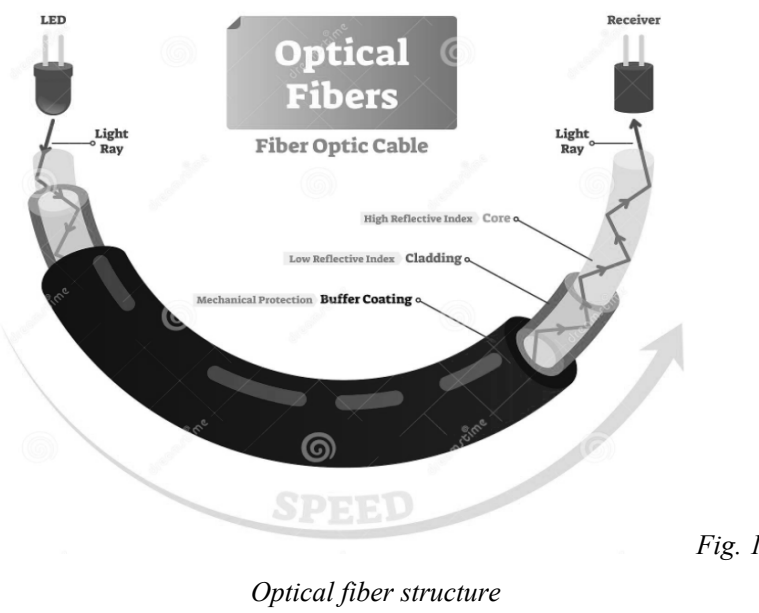

Among the widely used technologies that use light, as of now, it is optical fiber. Optical fiber is a dielectric waveguide, 
an insulated shielded light-conducting cable that operates on the phenomenon of internal reflection.

A. G. Bell in 1880 patented a photophone-a device for transmitting voice by means of a light signal with a selenium photodetector. The first commercial telephone systems were created only in 1977 and operated at a speed of $44.7 \mathrm{Mbit} / \mathrm{s}$. Single-mode fiber cables began to be produced in 1983 .

In 1990, Lynn Molliner (Bellcore) demonstrated the transmission of data at a speed of $2.5 \mathrm{Gbit} / \mathrm{s}$ over a distance of $7500 \mathrm{~km}$ (without intermediate signal amplifiers). In 1990, in the United States, the total length of optical fibers was about $9000000 \mathrm{~km}$.

In 2000, the total length of optical fibers in the United States alone exceeded 30 million kilometers. Fiber-optic communication lines operate in the frequency range $10^{13}-10^{16}$ $\mathrm{Hz}$, which is 6 orders of magnitude more than in the case of radio frequency channels (this provides a bandwidth of 50,000 Gbit / s). But the Earth's atmosphere is a poor medium for the propagation of light. For this reason, only the development of silicon fibers with a low absorption coefficient in the infrared range $(<0.2 \mathrm{~dB} / \mathrm{km})$ made it possible to widely distribute optical communication channels. $\sim 1000 \mathrm{~km}$ of fiber-optic cable is laid per day. Currently, the channels usually have a bandwidth of $\sim 1 \mathrm{Gbit} / \mathrm{s}$ and this is due to the limited speed of the equipment that converts the optical signal to electrical and vice versa. In the coming years, we should expect an increase in the performance of such devices by 100-1000 times. Given that $\Delta \mathrm{f}=\left(\mathrm{c} \Delta \lambda / \lambda^{2}\right)$, where $\mathrm{c}$ is the speed of light, $\mathrm{f}$ is the frequency, and $\lambda$ is the wavelength.

In 2002, Zonu developed a $1.25 \mathrm{Gbit} / \mathrm{s}$ phototransiver (GBIC) for transmitting and receiving data over the same fiber at a wavelength of $1310 \mathrm{~nm}$. For single-mode fiber, the transmission distance can be up to $10 \mathrm{~km}$. At a wavelength of $1550 \mathrm{~nm}$, a transmission distance of $40 \mathrm{~km}$ is achievable. A variant for $2.5 \mathrm{Gbit} / \mathrm{s}$ transmission speeds is being developed.

The fiber-optic connection guarantees a minimum of noise and high security (it is almost impossible to make a tap). Plastic fibers are applicable at connection lengths of no more than 100 meters and at limited speed $(<50 \mathrm{MHz})$. Recently (2006-7), plastic fibers have been developed that are suitable for transmission at a speed of $40 \mathrm{Gbit} / \mathrm{s}$ with a cable length of $30 \mathrm{~m}$ and at a speed of $5.35 \mathrm{Gbit} / \mathrm{s}$ with a cable length of 220 m (Lightware N4 2007). The probability of an error during transmission over an optical fiber is $<10^{-10}$, which in many cases makes it unnecessary to monitor the integrity of messages.

\section{SYSTEM COMPONENTS}

\section{A. GUI}

The user interface, in the field of industrial design, human - computer interaction is a space where interaction between humans and machines takes place. The purpose of this collaboration is to ensure perfect operation and control of the machine on the human side, and the machine simultaneously provides information that helps operators make decisions. Examples of this broad concept of user interfaces are: interactive aspects of computer operating systems, hand tools, operator controls for heavy equipment, and process controls. The design considerations used when creating user interfaces are related to areas such as ergonomics and psychology.

Generally, the goal of creating a user interface is to create a user interface that makes it easier (self-explanatory), efficient, and pleasant (user-friendly) to operate the machine in a way that provides the desired result. This usually means that the operator must make the slightest effort to achieve the expected result, as well as that the machine reduces undesirable results for humans. With the increasing use of personal computers and the relative decline in public awareness (interest) of heavy machines, the term "user interface" usually refers to a graphical user interface, while industrial control panels and equipment control mechanisms are more often referred to as human-machine interaction (HMI).

Other terms for the user interface are the human-machine interface (MMI), when the corresponding device is the humancomputer interface



Fig. 2. Main menu

The interface of the software system for decoding light codes was developed from the point of view of the main qualitative features of a good graphical user interface, namely [3]:

Clarity. The interface avoids ambiguity and makes everything clear through language, flow, hierarchy, and metaphors for visual elements.

Expressiveness. It's easy to make the interface transparent by over-refining and labeling everything, but this causes it to swell up when there are too many elements on the screen at the same time. If there are too many elements on the screen, it is difficult to find what one is looking for, and because of this, the interface becomes boring to use. The real challenge in creating a perfect interface is to make it concise and clear at the same time.

Introduction. Even if someone is using the interface for the first time, some elements may actually be familiar. Reallife examples can be used to pass the value.

Compliance. A great interface doesn't have to be sluggish. This means that it must provide the user with good 
feedback on what is happening and whether the user's input data is being processed successfully.

Sequence. It is important that your interface is consistent with your application, as it allows users to recognize usage patterns.

Aesthetics. Although you don't need to create an attractive interface so that it performs its task and does something well, but the time that users spend using your program will be more pleasant; and happier users can only do good things.

Performance. Time is money, and a cool interface should make the user efficient, with shortcuts and good design.

"Forgiveness." A good interface should not punish users for their mistakes, but should provide tools to fix them.

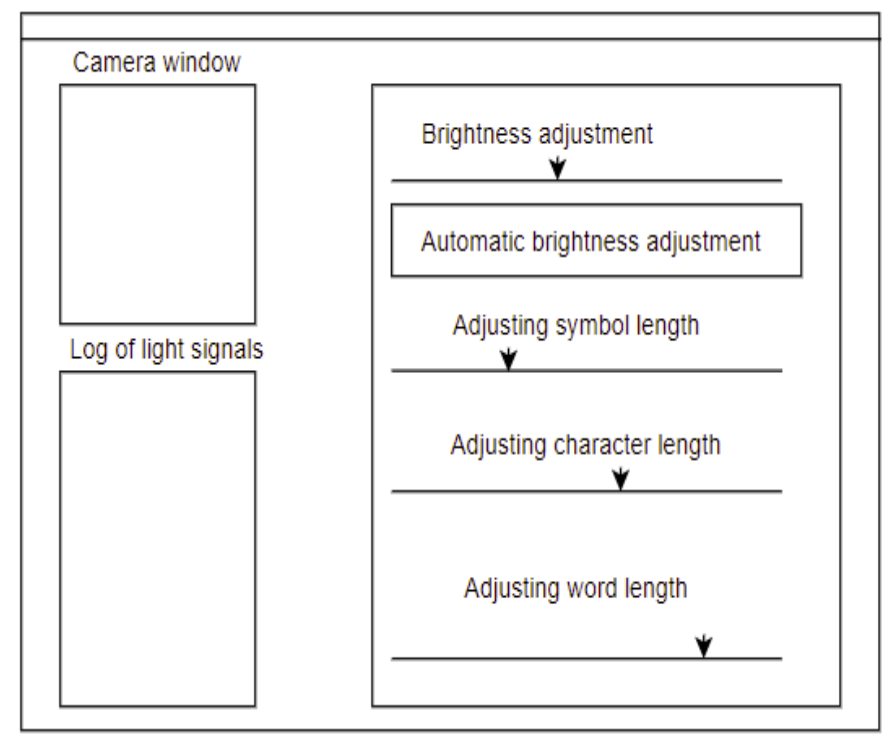

Fig. 3. Settings тепи

\section{B. LIGHT SIGNALS DECODER}

Incoming video is processed using Bitmap tools, which is a universal tool when working with an image, and the video is considered by the program as a set of frames, that is, a set of images.

First of all, you need to do two things:

- Draw a cross-shaped pointer for the user to point to the area of interest in the camera's field of view;

- Calibrate the program for the brightness of the source relative to the external environment.

After starting the camera, the user needs to select the point from which the light signal will be received in the video stream preview window. After clicking, a cross-shaped pointer will appear on the screen [4], the intersection of which will point to the selected point.

When everything is ready for execution, the user presses the start button and the program starts reading the incoming light stream. As soon as the program detects a flash, the received signals log receives information with the duration of each individual flash and attenuation. As in the case of calibration, the formula described in the YIQ model format documentation is used to obtain brightness [5], from which brightness ( $\mathrm{Y}$ ) is output as the sum of the products of RGB pixel values multiplied by constant coefficients:

$$
\mathrm{Y}=(0.299 * \operatorname{Red}+0.587 * \text { Green }+0.114 * \text { Blue })(1)
$$

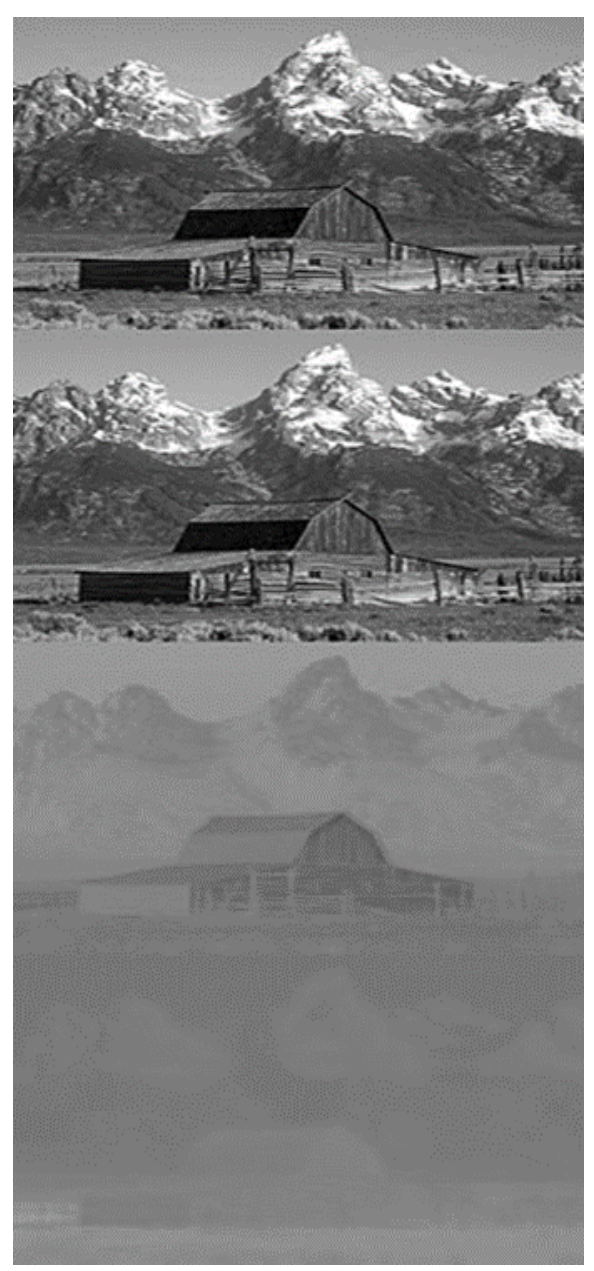

Fig. 4. Image with YIQ components separated

the result is divided by 36 to get the average brightness of a pixel, rather than a $6 \times 6$ square formed to improve decoding accuracy.

The system detects the flash and separately detects the time of the flash and its absence. This is done because, for example, Morse code was implemented in the system, where the role is played not only by the number of flashes, but also by their frequency, that is, the flash time and its absence.

Therefore, all decoded information in the form of Morse code is sent to the log of the Received message, where it can later be corrected and translated into the Latin alphabet.

\section{IV.FORMULATION OF THE PROBLEMS}

The main problem with modern data transmission is the need for compromise. If speed is required, they lay a cable network, if it is not possible to lay a cable network, they use cable - free or satellite technologies, but the speed and signal delay immediately suffer.

Advanced technologies are always created first for the military, then move to specific industries and only then enter 
the user market. The technology of cable-free optical communication has long been known and starting in 2019, NASA intends to use lasers to transmit data from space to Earth instead of radio channels [6]. One of the reasons for this transition is that it is less vulnerable to hackers and interception. In addition, the laser equipment has less weight and provides more bandwidth. The exchange rate will be 1.24 Gbit / s [7].

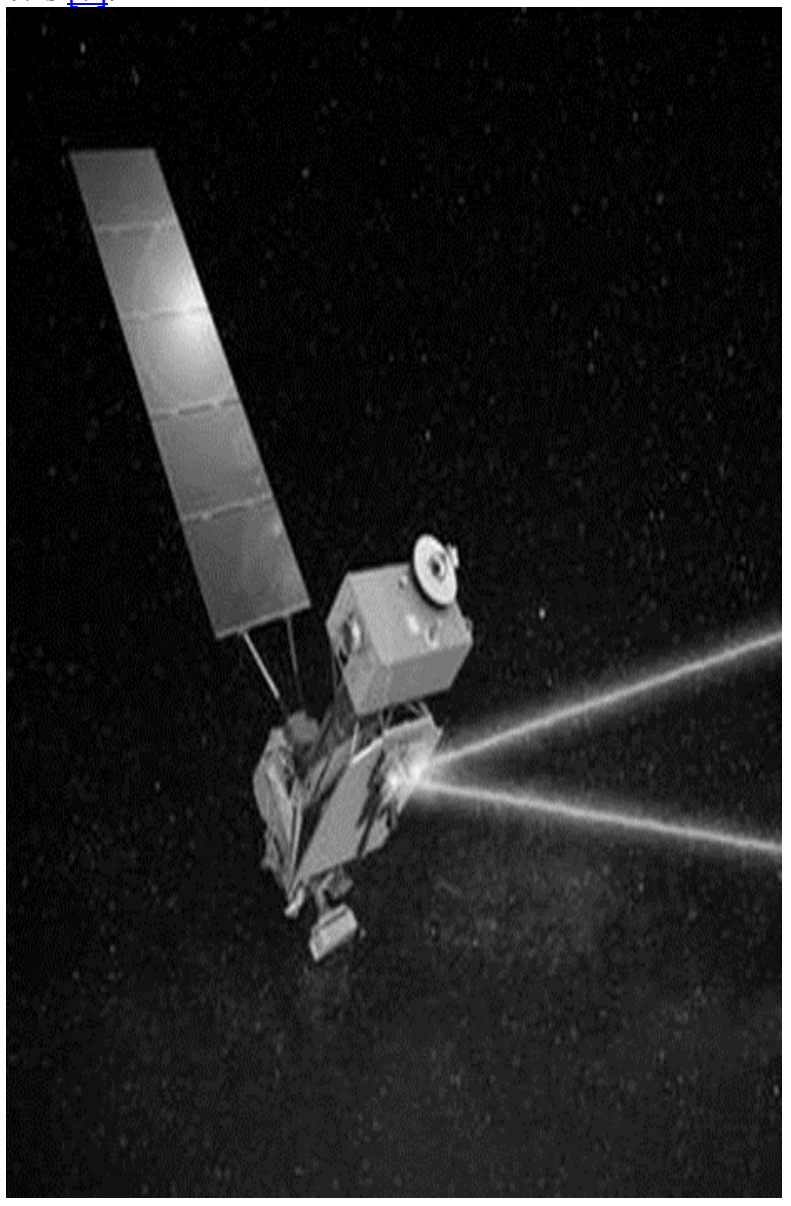

Fig. 5. NASA already using wireless optical system(LCR Laser Communication Relay) to communicate with near Earth stations

As of now, such systems are beginning to gain popularity, but due to the high cost and complexity of equipment, their distribution in the market is extremely small. The goal of the light code decoding software system is to simplify these systems for faster and easier assimilation in the market.

Let's take Wi-Fi systems as an example to express the problematics, so that it is one of the most popular and spread communication systems.

Transmitters operating on Wi-Fi frequencies are subject to registration. Although this procedure has recently been significantly simplified, it is still quite troublesome. Registration, along with the obvious inconveniences, has two advantages: firstly, you will not be subject to penalties, secondly - confidence in the absence of interference in your region. The latter is true only theoretically, since there are a huge number of unregistered stations and it is steadily growing in proportion to the decrease in the cost of wireless equipment. It should be recognized that the $2.4 \mathrm{GHz}$ band has become unregulated de facto.

Further. The well-known formula for calculating the total gain of the antenna-feeder path, used to calculate the maximum theoretically achievable speed, assumes an ideal state of the ether, an ideal antenna setup, and other ideal conditions that do not exist in nature. The formula does not take into account many factors, including:

- interference from other sources, in particular from transmitters operating at the same frequency, overtones from other RES, noise from power devices;

- multipath effects when passing through the foliage of trees, which are strongly dependent on the wind;

- influence of other obstacles;

- the difference between the parameters of the receiving and transmitting path, for example, the difference in the gain of the antennas, the quality of the circuitry, the number and nature of the filters.

To take into account the impact of these factors, you can perform a full spectrum analysis in the area with the appropriate equipment or assign this work to specialists. But since this pleasure is not cheap, in practice, after installation, they simply go through all the channels of the range (for Europe/Russia 13 channels from 2.412 to $2.472 \mathrm{GHz}$ are allocated).

Many of these difficulties can be overcome by purchasing more powerful antennas and amplifiers and minimizing the loss between the wireless device and the antenna by reducing the length of the cable and the number of joints. It is possible (although risky) to abandon the lightning protection.

The use of more powerful antenna-feeder devices almost always solves the problem of poor or unstable communication, and in some cases allows to establish communication even in the absence of a line of sight, due to diffraction and reflection (although one should not count on this and should always try to lay a wireless path in the line of sight between the nodes).

\section{FUNCTIONS AND FUNCTIONAL PRINCIPLES OF THE SOFTWARE SYSTEM OF DECODING LIGHT CODES}

The software system for decoding light codes is not overloaded with functionality [8], because the simpler the mechanism, the more reliable and efficient it is. The program consists of several key modules:

- Module for selecting a video recording device from the available host machine;

- Module for selecting the area of interest in the camera's field of view;

- Morse code translation module-Latin and reverse;

- Morse code light signal decoding module;

- User interface with controls.

The system also contains a block of settings that contain Morse code light delay values and calibrate the brightness of the light source. Previously, the software system sets the most popular delays for Morse code, so that the program is as ready to work as possible immediately after installation. To calibrate the brightness, there is a manual adjustment option and an 
auto-calibration button that automatically detects the brightness of the source in the area of interest in the camera's field of view.



Fig. 6. Principles of work of the software system of decoding light codes

\section{COMPARATIVE ANALYSIS OF EXISTING WIRELESS OPTICAL COMMUNICATION SYSTEMS}

As such, this implementation has no analogues in the usual sense, since it is a software product, however, as in the situation with optical fiber, using hardware solutions, specialized equipment, the selection of related solutions is slightly expanded. Currently, there are several implementations of cable-free channels. Table 1 [9].

Table 1

The example of optical receiving-sending equipment characteristics

\begin{tabular}{lll}
\hline \hline & LED-LINK 300 & $\begin{array}{l}\text { LaserLink 4E1 / } \\
300\end{array}$ \\
\hline $\begin{array}{l}\text { Recommended } \\
\text { distance [m] }\end{array}$ & $<300$ \\
Bandwidth [Mbps] & $2-43$ & $4 * 2.048$ \\
BER & $\leq 10-9$ & $\leq 10-6$ \\
Sender & IP-LED & IP-LED \\
Sended power [mW] & $50 / 60$ & $50 / 60$ \\
Beam divergence & $<10$ & $<10$ \\
[mrad] & & \\
$\begin{array}{l}\text { Dynamic range [db] } \\
\text { Network interface }\end{array}$ & $>30(1: 1000)$ & $>40(1: 10000)$ \\
Fiber diameter & $50-60 / 120$ & - \\
[mcm] & & \\
Wave length (RX) & $780 . .900$ & - \\
[nm] & & \\
Wave length (TX) & 850 & - \\
[nm] & & $-20 \div+503$ \\
Working temperature & $-20 \div+50$ & $<300$ \\
[ o C ] & & \\
Recommended & $<300$ & \\
distance [m] & & \\
Bandwidth [Mbps] & $2-43$ & \\
\hline \hline
\end{tabular}

VCSEL (Vertical Cavity Emitting Laser) - emits a laser with a vertical resonator. The above - mentioned lasers (CBLMunster, Germany) belong to class $1 \mathrm{~m}$ (safe for the eyes).

The most common data transmission channels today are those with speeds from 10 Mbit / s to 1-2.5 Gbit / s at distances from $100 \mathrm{M}$ to $4 \mathrm{~km}$. Options for using such channels for communication with airliners are being discussed, since this does not create problems with navigation, but nothing has been known about the implementation of such projects yet.

Fundamentally new opportunities were opened up by the discovery of infrared lasers. The laser generates a weakly scattered beam of light in the air (about $1 \mathrm{~mm}$ in diameter).
This makes it possible to transmit an open beam over a relatively long distance (up to $10 \mathrm{~km}$ ). But the same property of the Beam also creates certain problems. In the atmosphere, convection flows of hot air rise up from hot objects, which vary the refractive index. Many people have observed this phenomenon over the highway on a hot summer day, when the car in front seems to take off from the ground and soars in a swayed haze. The same phenomenon underlies the appearance of Mirages. It should also be taken into account that the sun creates a radiation flux in the infrared region no less than in the visible region. Optical channels involve the use of two parallel beams, one for each transmission direction. The diameter of the sensitive surface of the detector usually does not exceed $1 \mathrm{~mm}$.

However, light, as a storage medium, has certain features in use. The main and almost the only significant feature of using a light signal transmission system is the poor conductivity of light electromagnetic waves in the Earth's atmosphere. Therefore, the main task in the development and deployment of such systems is to avoid and/or level the impact of adverse factors in the atmosphere.

As it was described above, one of the critical factors is convective waves [10].

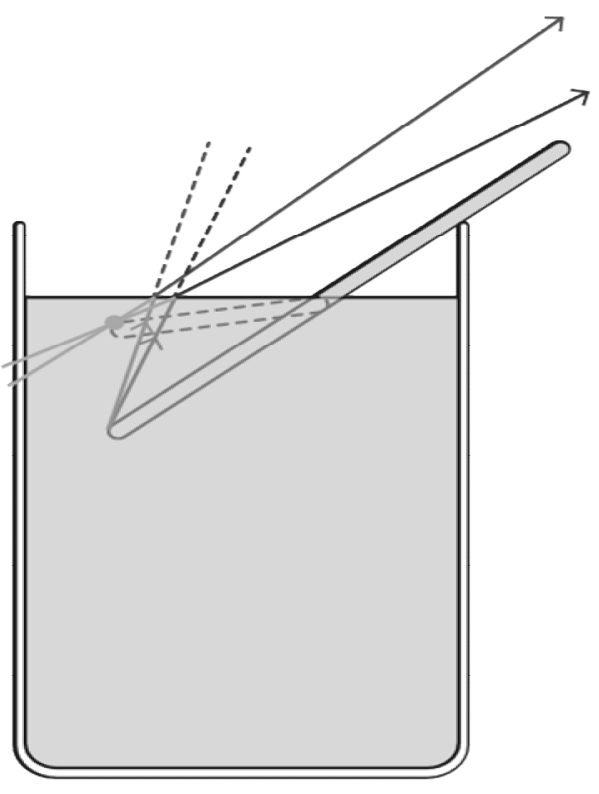

\section{Fig. 7. Light going through convective air acts like light moving though liquids}

To eliminate the influence of convective air flows from the heated roof surface, defocusing beams is usually used, so that even if the beam axis deviates, the light spot does not fall into the sensitive area of the detector. This method assumes that there is an excess of the light power of the transmitting laser [11].

For this reason, as well as due to the absorption of the beam by rain and fog, communication channels with an open laser beam are not widely used. But sometimes, due to the lack of necessary cable channels, or due to the objections of telephone companies, an open beam can be useful for organizing communication between not very remote buildings. 
An open beam provides a fairly high level of security, since to intercept messages, you need to "reach" the beam. And it is not so easy to detect an infrared beam without special tools.

When designing such communication channels, it is necessary to take into account the attenuation of the signal a geom $[\mathrm{DB}]$ associated with the beam geometry:

$$
\text { a geom }=20 \log \{\text { a geom } \times \mathrm{R} / \mathrm{d} \text { ent }\} \mathrm{DB}(2)
$$

where $\mathrm{a}$ - geom is the angle of divergence in radians, $\mathrm{R}$ is the transmission distance in meters, and $\mathrm{dBX}$ is the diameter of the entrance window in meters. Attenuation associated with absorption and scattering must also be taken into account:

$$
\text { a dispersed }=(17 / \mathrm{S})\{0,55 / 10,195 \mathrm{~S} / \mathrm{km}\}[\mathrm{DB} / \mathrm{km}](3)
$$

where a diffused is attenuation in decibels per kilometer, 1 is the radiation wavelength in microns, and $\mathrm{S}$ is the visibility range $[\mathrm{km}]$. I must say that the range and reliability of transmission in this case strongly depends on weather conditions, as it can be seen in Fig. 8. For that, such factors as fog or rain can intrude into the process of data transmission and cause errors or data loss.

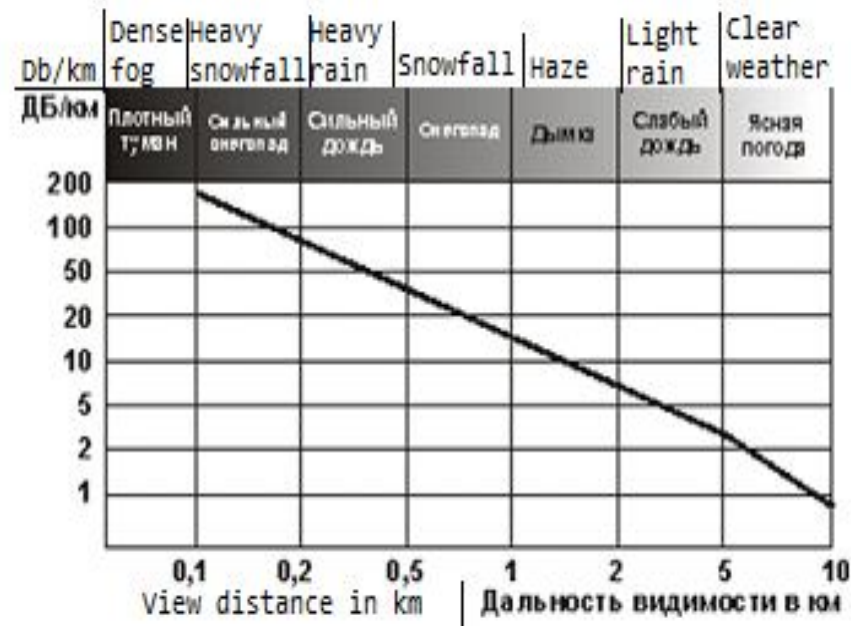

Fig. 8. Depending of light beam attenuation on atmosphere state

But for communication within halls with large volumes, this option is quite attractive. It uses special defocusing systems and reflexes from walls and other objects. This scheme is also attractive from the point of view of security. After all, unlike radio waves, infrared radiation cannot pass through walls and even glass. A local area network built on this principle is a single-segment LAN.

But the Earth's atmosphere is a poor environment for light propagation. For this reason, only the development of Silicon fibers with a low absorption coefficient in the infrared range $(<0.2 \mathrm{DB} / \mathrm{km})$ made it possible to widely use fiberoptic communication channels. $\sim 1000 \mathrm{~km}$ of fiber optic cable is laid per day. Currently, channels usually have a bandwidth of 1-100 Gbit / s. In the coming years, we should expect an increase in the performance of such devices by 10-100 times. Considering that

$\Delta \mathrm{f}=\left(\mathrm{c} \Delta \lambda / \lambda^{2}\right)$, where $\mathrm{C}$ is the speed of light, $\mathrm{f}$ is the frequency, and $\lambda$ is the wavelength, for the most popular range $\lambda=1300 \mathrm{Nm}$ and $\Delta \lambda=0.17 \mathrm{~m}$, we have $\Delta \mathrm{f}=\sim 30$ thz.

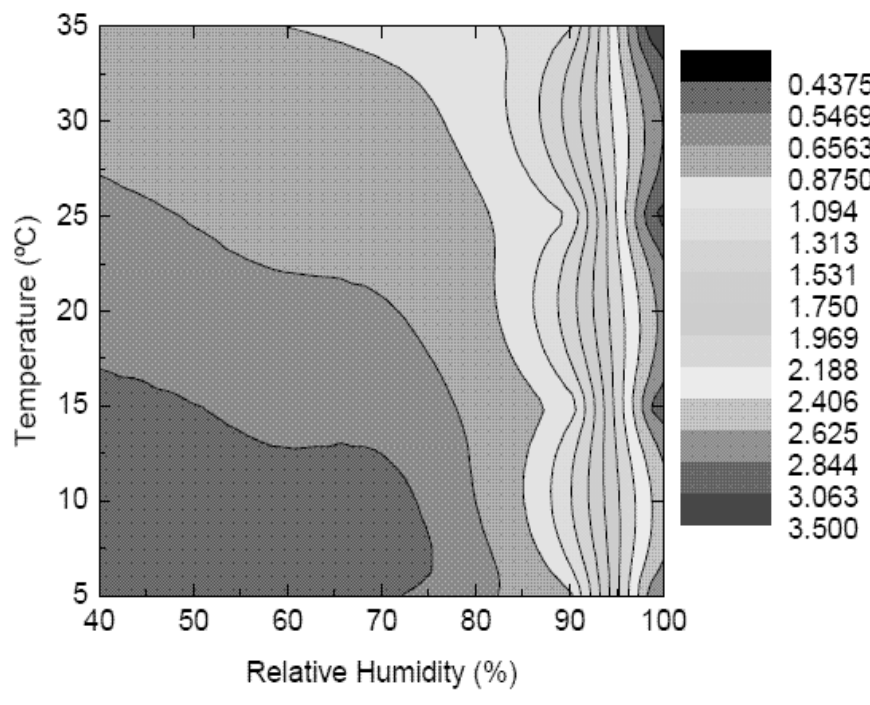

Fig. 9. Depending of light beam absorption on humidity and temperature of the air

\section{CONCLUSIONS}

The software system for decoding light codes is one of the most promising technologies of our time. It is increasingly gaining popularity due to its obvious advantages. Enterprises that implement this technology will receive significant savings on capital costs and current operating costs due to cable-free data transmission, voice, and the complexity of the light beam as an information carrier will increase the level of security and eliminate external interference, and additional energy costs will be decreased by reducing the amount of equipment. Implementing a cable-free optical communication system will allow organizations to reduce infrastructure investment from the data center to the end user, significantly reducing the number of cable routes. The overall result is a dramatic reduction in costs and network complexity. As a rule, the introduction of such technology significantly expands the standard network lifecycle.

The result of testing the system showed a good result: passing the test was $95 \%$. 5\% of non-completed tests were tests of the actual decoding of the light signal. The reason was poor lighting, which affected the test, due to the features of the hardware component of the test Assembly, namely, the low aperture of the test camera.

\section{References}

[1] Komine, T. and Nakagawa, M., (2004). Fundamental analysis for visible-light communication system using LED lights. IEEE Transactions on Consumer Electronics, 50(1), pp.100-107;

[2] Price Hossell, K., (2002). Morse code. Chicago, Ill.: Heinemann Library ;

[3] Stephens, R., (2010). C\# Graphics Programming. Hoboken: Wrox [Imprint] ;

[4] Chandrasekara, C., n.d. Hands-on functional test automation;

[5] Li, K. and Wu, M., (2006). Effective GUI Testing Automation. Hoboken: John Wiley \& Sons ;

[6] Patrick Nelson, NASA to use data lasers to beam data from space to Earth, Network World, Aug 30, 2018;

[7] H. Le Minh, D. O'Brien, G. Faulkner, L. Zeng, K. Lee, D. Jung, Y. Oh, and E. T. Won, " $100-\mathrm{Mb} / \mathrm{s}$ NRZ visible light 
communications using apostequalized white LED," IEEE

Photon. Technol. Lett., vol. 21, no. 15,pp. 1063-1065, 2009;

[8] IEEE Standard for Local and Metropolitan Area NetworksPart 15.7 : Short-Range Wireless Optical Communication Using Light, IEEE Std. 802.15.7-2011, 2011;

[9] CBL Communicaton by light. (2021). LaserLink 4E1. [online] Available at: https://www.cbl.de/en/products/laser-link4e1/laserlink-4e1;

[10] Book.Itrep.ru. (2021) Fiber optic channels and wireless optical communiucations. [online] Available at: http://book.itep.ru/3/optic_32.htm] ;

[11] Zhang, J., n.d. Controlling light with photonic metamaterials.



Vladyslav Barsiienko is a fourth-year computer engineering student at Lviv Polytechnic National University.

His research interests include image processing, wireless networks and cyber security.

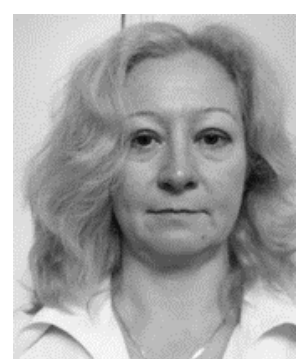

Oksana Lashko was born in 1976 in Lviv, Ukraine. She received the B.S. and M.S. degree in Lviv Polytechnic State University, Lviv, in 1999. From 1999 to 2002, she was postgraduate at Lviv Polytechnic National University. Since 2007, she is a senior lecturer at the Computer Engineering Department of Lviv Polytechnic National University. Her research interests include the development of signal processing tools at the algorithmic and software levels, research of image encoding and compression problems.. 